\title{
FAULT DIAGNOSIS METHOD OF ROLLING BEARING BASED ON DEEP BELIEF NETWORK
}

\author{
Wenfeng Zhang ${ }^{1}$ \\ ${ }^{1}$ Department of Mechanical Engineering, Shanghai University of Engineering Science, Shanghai, 201600, China
}

\begin{abstract}
A fault identification method of rolling bearing based on depth belief network is proposed, which does not need to extract fault features in advance. Vibration signal is directly used as the input of the whole system. Fault feature extraction and fault identification can be automatically accomplished by using the powerful feature extraction ability of deep confidence network. The results show that the proposed method is able to not only adaptively mine available fault characteristics from the data, but also obtain higher identification accuracy than the existing methods.
\end{abstract}

Keywords: - deep belief network; RBM; feature extraction; vibration signal; fault diagnosis

\section{INTRODUCTION}

With the development of science and technology, the electromechanical equipments in aviation, aerospace, industry and other fields are becoming more and more complex, intelligent and comprehensive. Moreover, their operating conditions and working environment are becoming more and more complex and changeable, resulting in higher and higher maintenance and safeguard costs. At the same time, due to the increase of components and influencing factors, the probability of failure is increasing. Therefore, accurate and effective fault diagnosis of complex equipment system becomes an effective way to improve system security and reliability and reduce maintenance costs.

Traditional intelligent methods are also called shallow learning models, including artificial neural networks, support vector machines, LR and so on. Although these methods have made great achievements in intelligent fault diagnosis, they still can not use less prior knowledge to analyze complex faults. Because of poor performance and generalization ability, it is difficult for them to express complex functions. These facts demonstrate the limitations of shallow networks and encourage people to explore how to extract features and represent complex functions through deep networks.

In recent years, deep learning, as an emerging method in the field of machine learning, has achieved brilliant results in the fields of image and speech recognition with its powerful automatic feature extraction capability. As one of the classical algorithms for deep learning, Deep Belief Network successfully solves problems such as information retrieval, dimension reduction, fault classification and so on with its excellent feature extraction and training algorithms. Compared with the traditional fault diagnosis method, the DBN method has the following advantages: 1) Deep learning has powerful feature extraction ability, which can automatically extract features from a large amount of data, reducing the dependence on expert fault diagnosis experience and signal processing technology. It reduces the uncertainty of feature extraction and fault diagnosis caused by manual participation in traditional methods; 2) By establishing a deep model, it can well represent the complex mapping relationship between signal and health status, which is very suitable for big data background. Diagnostic and analytical needs for diversity, nonlinearity, and high-dimensional health monitoring data. Therefore, applying deep learning to the field of fault diagnosis has certain timeliness, practicability and versatility.

Aiming at the problem of traditional rolling bearing fault diagnosis, this paper proposes a fault feature extraction and diagnosis method based on deep confidence network. The method directly extracts fault characteristics and health status from the original time domain signal.

\section{THEORETICAL FRAMEWORK}

Deep Belief Network is formed of many RBMs, it is a deep learning model effective for sequential data processing.

\subsection{Architecture of RBM}

The RBM model consists of two layers: one is the input layer, also known as the visible layer, and the other is the output layer, also known as the hidden layer. RBM can be expressed as a binary undirected graphical model. All visible elements of the RBM are fully connected to the hidden unit, and the units within the layer do not have any connection to each other. That is, there is no connection between visible cells or between hidden cells. The architecture of the RBM is shown in Figure 1. 
In Figure 1, $v$ represents the visible layer, $i$ is the $i$ th visible unit, $h$ is the hidden layer, and $j$ is the $j$ th hidden unit. Connections between these two layers are undirected. An energy function is proposed to describe the joint configuration $(v, h)$ between them, which is expressed as

$$
E(v, h)=-\sum_{i=1}^{I} a_{i} v_{i}-\sum_{j=1}^{J} b_{j} h_{j}-\sum_{i j} v_{i} h_{j} w_{i j}
$$

Here, $v_{i}$ and $h_{j}$ represent the visible unit $i$ and hidden unit $j$ respectively; $a_{i}$ and $b_{j}$ are their biases. $w_{i j}$ denotes the weight between these two units. Therefore, the joint distribution of this pair can be obtained using the energy function where $\mathrm{h}$ is the model parameter set containing $a, b$, and $w$ :

$$
\begin{aligned}
& p(v, h)=\frac{1}{Z(\theta)} \exp (-E(v, h)) \\
& Z(\theta)=\sum_{v} \sum_{h} \exp (-E(v, h))
\end{aligned}
$$

Due to the particular connections in RBM model, it satisfies conditional independent. Therefore, conditional probability of this pair of layers can be written as:

$$
\begin{aligned}
& p(h \mid v)=\prod_{i} p\left(h_{i} \mid v\right) \\
& p(v \mid h)=\prod_{j} p\left(v_{j} \mid h\right)
\end{aligned}
$$

Mathematically,

$$
\begin{aligned}
& p\left(h_{j}=1 \mid v\right)=\sigma\left(b_{j}+\sum_{i} v_{i} w_{i j}\right) \\
& p\left(v_{i}=1 \mid h\right)=\sigma\left(a_{i}+\sum_{j} h_{j} w_{i j}\right)
\end{aligned}
$$

where $\sigma(x)$ is the activation function. Generally,

$$
\sigma(x)=\frac{1}{1+\exp (-x)} \text { is adopted. }
$$

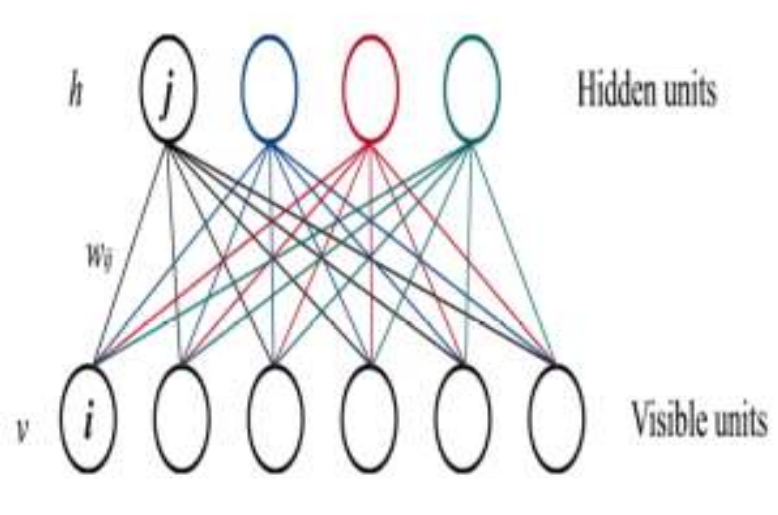

Fig 1: Architecture of RBM

\subsection{DBN Architecture}

The DBN model is a deep network architecture with multiple hidden layers and many non-linear representations. It is a probability generation model that can be formed by RBM, as shown in Figure 2. It illustrates the way in which one RBM is stacked on top of another. The DBN architecture can be built by stacking multiple RBMs one by one to form a deep network architecture.

As DBN has multiple hidden layers, it can learn from the input data and extract hierarchical representation corresponding to each hidden layer. Joint distribution between visible layer $v$ and the $l$ hidden layers $h_{k}$ can be calculated mathematically from conditional distribution $P\left(h^{k-1} \mid h^{k}\right)$ for the $(k-1)$ th layer conditioned on the kth layer and visible-hidden joint distribution $P\left(h^{n-1}, h^{n}\right)$ :

$$
P\left(v, h^{1}, \ldots, h^{n}\right)=\left(\prod_{k=1}^{n-1} P\left(h^{k-1} \mid h^{k}\right)\right) P\left(h^{n-1}, h^{n}\right)
$$

For classification tasks, fine-tuning all the parameters of this deep architecture together is needed after the layer-wise pre-training. It is a supervised learning process using labels to eliminate the training error and improve the classification accuracy. 


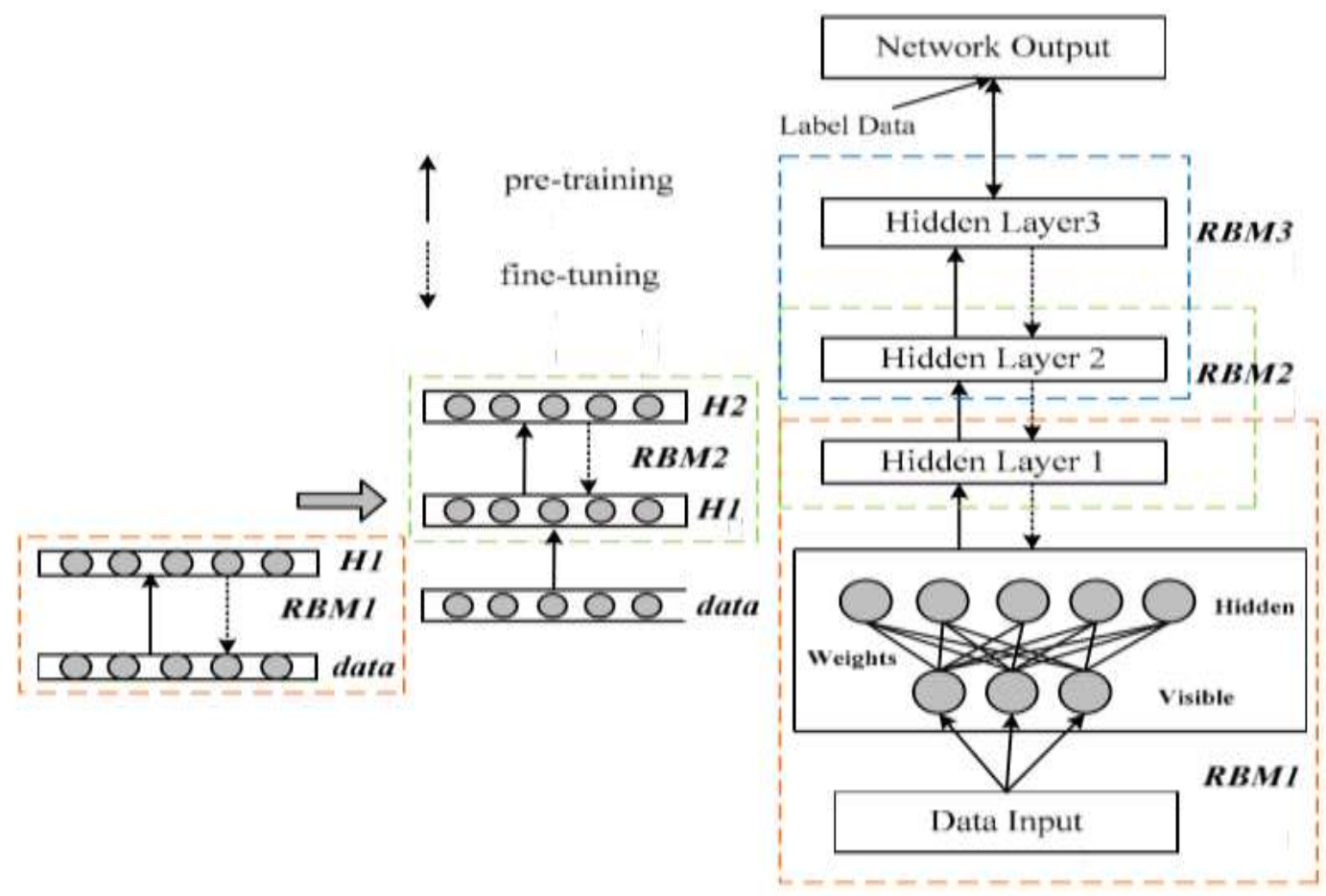

Fig 2: The general model of Deep Belief Network.

\section{DBN FAULT FEATURE EXTRACTION}

\section{PROCESS}

Firstly, the DBN model is unsupervised layer by layer, and then the DBN model is supervised and trained using the inverse fine-tuning algorithm. Finally, the data set to be tested is input into the trained DBN model, and the output vector of each hidden layer is recorded. The fault feature extraction steps are shown in Table 1.

Table 1: DBN Feature extraction process

\begin{tabular}{|l|l|}
\hline Step & Process content \\
\hline 1 & Define fault diagnosis and fault types \\
\hline 2 & Normalize the [ 0,1] of the fault signal \\
\hline 3 & Dividing datasets into training sets and test sets \\
\hline 4 & Initialize DBN related parameters \\
\hline 5 & Training Stacking RBM with Training Sets \\
\hline 6 & $\begin{array}{l}\text { The test set is entered into the trained stacked } \\
\text { RBM model and the output vector of each } \\
\text { hidden layer is recorded }\end{array}$ \\
\hline
\end{tabular}

In order to visually see the effect of the DBN method extracting features from the original data, it is necessary to visualize the extracted features. Since the original data and the feature dimensions extracted by each layer of the DBN are relatively high and cannot be directly observed, the PCA method is used to reduce Data dimension to make it easier to see the feature extraction capabilities of the DBN method.

\section{ROLLING BEARING DATA SET}

As a key part of major mechanical equipment, the bearing's normal working condition is directly related to the performance of the whole equipment. Therefore, it is of practical significance to diagnose the bearing in major mechanical equipment. The ability to extract and diagnose fault features from the original signal using the DBN model was further verified by bearing fault diagnostic experiments.

\subsection{Dataset Description and Fault Type Definition}

The data set used in this part of the experiment is the bearing data set collected by the Electronic Engineering Laboratory of Case Western Reserve University. This data set was acquired at 4 different loads ( 0 to $3 \mathrm{hp}$ ) at a sampling frequency of $12 \mathrm{kHz}$. This bearing test system simulates the normal state of the bearing, the outer ring fault, the inner ring fault and the ball fault, and each fault type has 3 fault depths. Three data sets A, B and C were constructed with experimental data from 0 to $2 \mathrm{hp}$ under 3 loads, each containing 10 fault categories. Using the window containing 2048 points to divide the acquired raw vibration signals, 50 samples can be obtained for each fault category. Considering the actual situation, the same fault category may correspond to multiple loads, and the data set A to $\mathrm{C}$ is combined to obtain the data set $\mathrm{D}$. The statistics of these four data sets A to D are shown in Table 2, where Normal represents the normal state of the bearing, RF, IF, and $\mathrm{OF}$ represent the ball, bearing inner and outer ring faults, respectively, and RF13 represents different fault depths. 
Table 2: Description of bearing fault datasets

\begin{tabular}{|l|l|l|l|l|}
\hline Fault category & Fault depth/mm & Data Set A/B/C & Data Set D & Fault category \\
\hline Normal & 0 & 600 & 1800 & 1 \\
\hline RF1 & 0.18 & 600 & 1800 & 2 \\
\hline RF2 & 0.36 & 600 & 1800 & 3 \\
\hline RF3 & 0.54 & 600 & 1800 & 4 \\
\hline IF1 & 0.18 & 600 & 1800 & 5 \\
\hline IF2 & 0.36 & 600 & 1800 & 6 \\
\hline IF3 & 0.54 & 600 & 1800 & 7 \\
\hline OF1 & 0.18 & 600 & 1800 & 8 \\
\hline OF2 & 0.36 & 600 & 1800 & 9 \\
\hline OF3 & 0.54 & 600 & 1800 & 10 \\
\hline
\end{tabular}

\subsection{Experimental Results}

First, the original data is linearly normalized and transformed so that its range is limited to $[0,1]$. The original data mentioned later are normalized data. The DBN model uses a classic 5-layer model with a number of nodes of 2048-1200-600-300-10. The weight of the DBN model is initialized with a normal random distribution, and the threshold of the DBN model is initialized to 0 . The maximum number of iterations of RBM is 100 , the learning rate is 0.1 , and the momentum parameter is 0.9. Sixty percent of each failure category sample was randomly selected for training and the remainder was tested. Among the four data sets $A$ to $D$, the data sets $A$ to $C$ have the same property, that is, they are collected under a single load, so the data sets A to $\mathrm{C}$ are put together for analysis of the results. Due to space limitations, this paper only gives the visualization results of fault feature extraction of dataset $\mathrm{A}$ using DBN model, as shown in Figures 3-4.

It can be seen from Figures 3 to 4 that the DBN can perform better feature extraction on data sets with different fault depths and fault types under a single load. Considering the actual situation, the same fault may be generated under different loads. Under this consideration, data set $\mathrm{D}$ is constructed. Figure 5 shows the original feature visualization result of data set D, and Figure 6 shows the use of DBN. The model visualizes the fault feature extraction of data set $\mathrm{D}$.

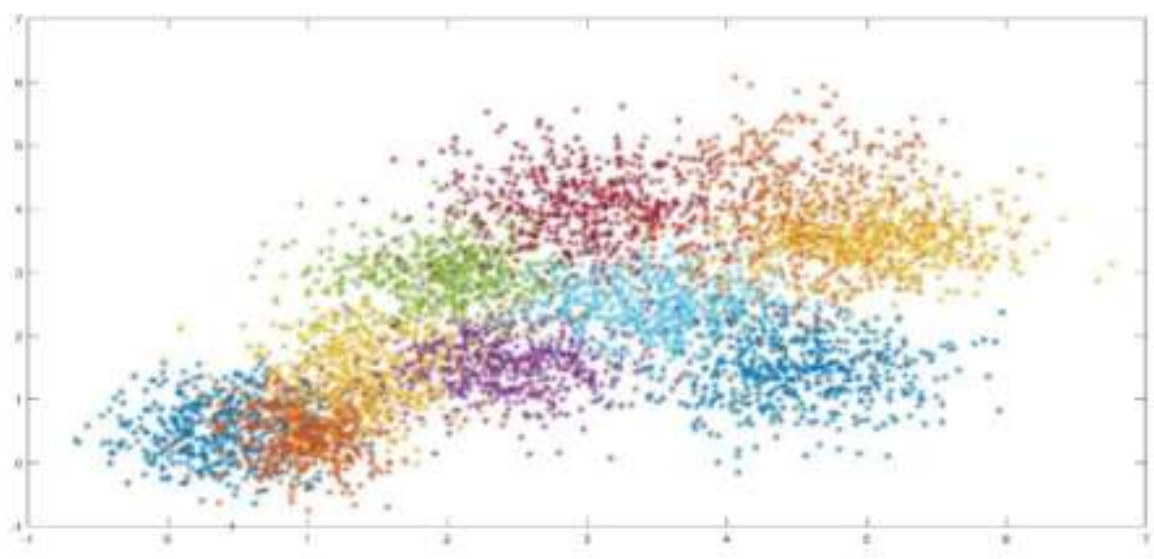

Fig 3: Visualization of the raw features in dataset A 


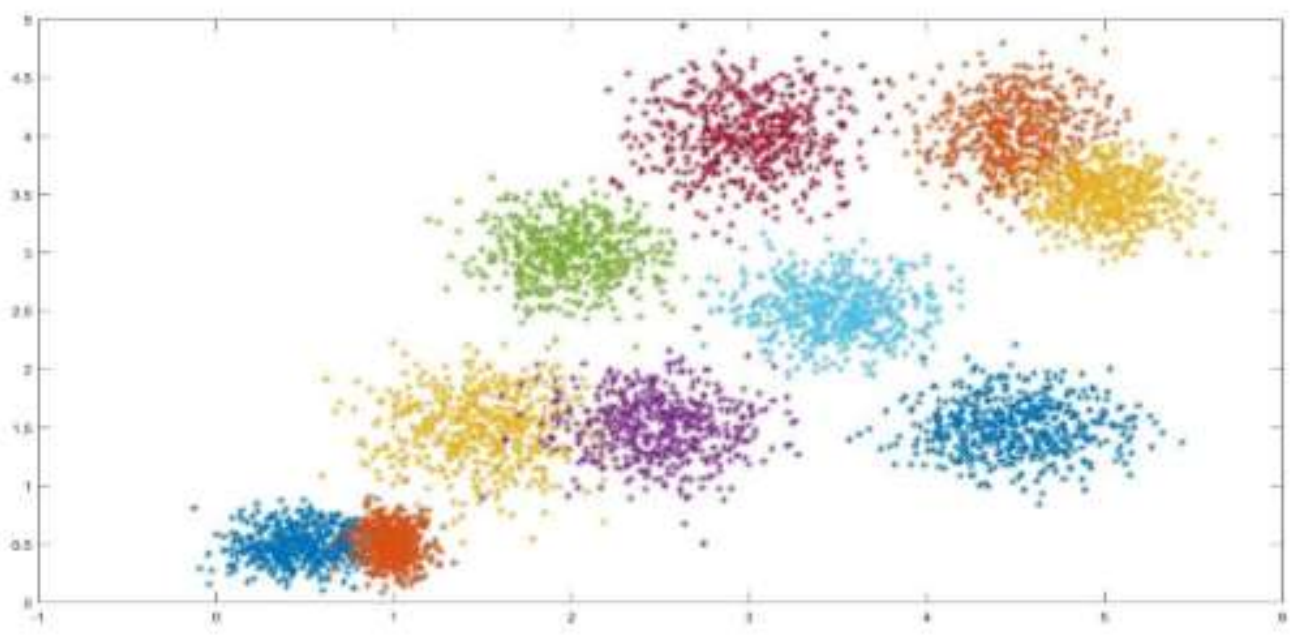

Fig 4: Features visualization of the third hidden layer in dataset $\mathrm{A}$

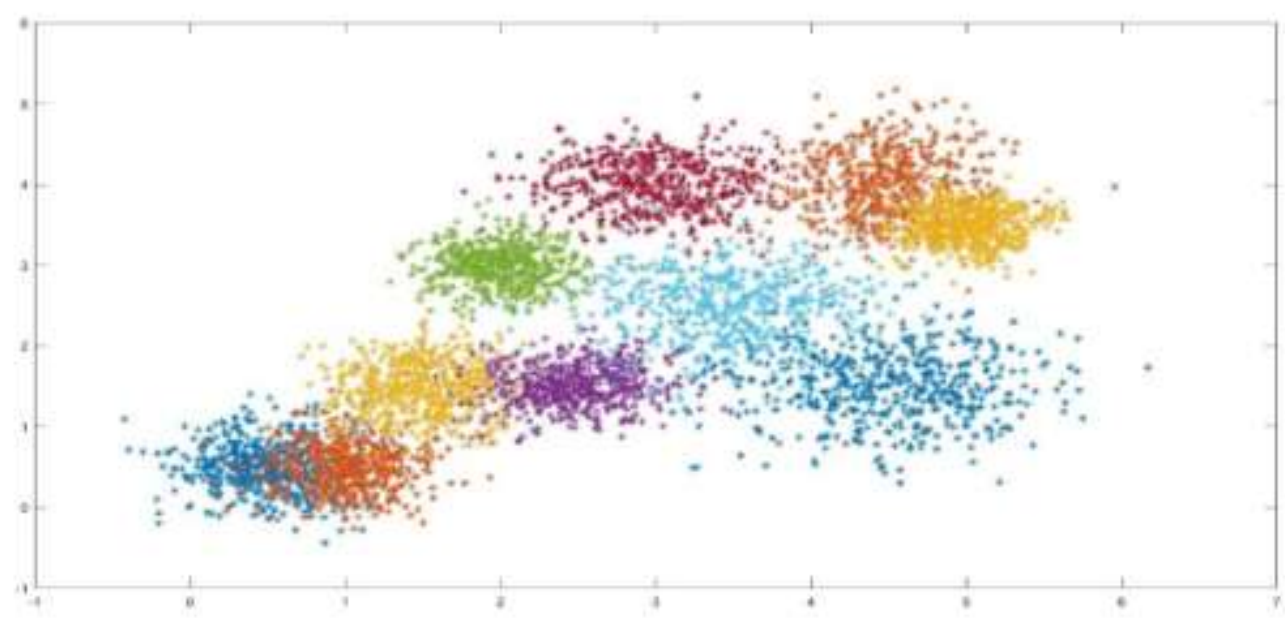

Fig 5: Features visualization of the raw features in dataset D

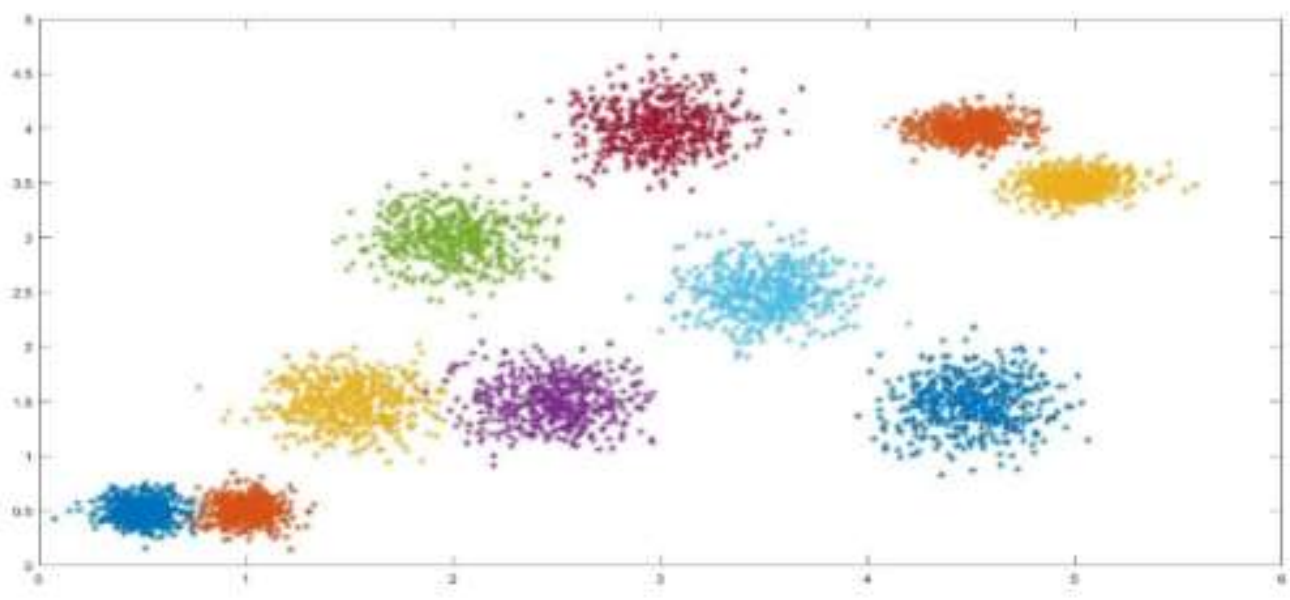

Fig 6: Features visualization of the third hidden layer in dataset D 
As can be seen from Figure 5, only two types of faults are distinguished, and the remaining eight faults are tightly clustered together. It can be seen from Figure 6 that after the features are extracted layer by layer through the DBN model, most of the faults can be directly separated, and the 10 fault categories are in an outward diverging state. Although there will be slight overlap, the whole can be distinguished. open. Combining the results in Figures 5 and 6 , it can be clearly seen that there is less overlap in Figure 6, and the whole is a divergent state, which is more conducive to distinguishing by the classifier. Therefore, the DBN model can not only extract fault features under a single working condition, but also extract fault features under various working conditions.

In order to evaluate the fault identification capability of the DBN model, the faults are further identified and classified based on the fault feature extraction. The method proposed in this paper is compared with the traditional fault diagnosis method, and the comparison results are shown in Table 3. The classification accuracy of DBN in Table 3 is the result of 10 averages, that is, 10 times of repeated operation for each data set, and the average of the accuracy of the failure classification is taken 10 times.

Table 3: Diagnostic results of bearing datasets

\begin{tabular}{|l|l|l|l|l|}
\hline Method & Data set A & Data set B & Data set C & Data set D \\
\hline $\begin{array}{l}\text { Wavelet leaders multifractal } \\
\text { feature+SVM }\end{array}$ & 89.1 & & & \\
\hline SS-DPSOM & & & & 95.8 \\
\hline Time+MLP & 95.7 & 99.6 & 99.4 & \\
\hline PSO-WKLFDA & 97.2 & & & \\
\hline OAO-PSO-WKLFDA & 98.8 & & & \\
\hline Frequency Domain+DBN & 99.9 & 99.6 & 99.7 & 99.6 \\
\hline Time Domain+DBN & 100 & 100 & 100 & 99.4 \\
\hline
\end{tabular}

Based on the results in Table 3, it is shown that the DBN model has a large advantage in fault feature extraction and diagnosis directly from the original data. For the data set D which is closer to the actual situation, the DBN method also obtains higher fault diagnosis accuracy, which fully demonstrates that the method of fault diagnosis using the DBN model from the original signal can adapt to complex working conditions and accurately identify the fault location and fault degree.

\section{CONCLUSION}

In this paper, a fault bearing feature extraction and diagnosis method for rolling bearing is presented. The method is based on the deep learning framework and integrates fault feature extraction and classification into a model. The effectiveness of the proposed method is verified on the simulation dataset and bearing dataset. The results show that: 1) The DBN model can extract fault features layer by layer from the original time domain signal and obtain higher diagnostic accuracy; 2) and tradition Compared with the feature extraction method, the proposed method still achieves higher classification accuracy than the traditional method without relying on signal processing technology and expert experience. 3) The method is not limited to the periodicity of the signal, and the fault diagnosis method is enhanced. Versatility and practicality. Due to the time relationship, the method used in this paper only considers the case of a single signal. In reality, for each diagnostic system, multiple sensors will collect data simultaneously, and how to use the deep learning method to effectively use the multi-source heterogeneous sensor data. Fault diagnosis of complex systems is the direction that needs further research in the future.

\section{REFERENCES}

[1] H Gao, L Liang, X Chen, et al. Feature extraction and recognition for rolling element bearing fault utilizing short-time Fourier transform and non-negative matrix factorization. Chinese Journal of Mechanical Engineering, 2015, 28(1): 96-105.

[2] J Antonino-Daviu, S Aviyente, E G Strangas, et al. Scale invariant feature extraction algorithm for the automatic diagnosis of rotor asymmetries in induction motors. IEEE Transactions on Industrial Informatics, 2013, 9(1): 100-108.

[3] Y Wang, F Zhang, T Cui, et al. Fault diagnosis for manifold absolute pressure sensor (MAP) of diesel engine based on Elman neural network observer. Chinese Journal of Mechanical Engineering, 2016, 29(2): 386-395.

[4] M H Drif, A J Cardoso. Stator fault diagnostics in squirrel cage three-phase induction motor drives using the instantaneous active and reactive power signature analyses. IEEE Transactions on Industrial Informatics, 2014, 10(2):1348-1360.

[5] G Chen, L Qie, A Zhang, et al. Improved CICA algorithm used for single channel compound fault diagnosis of rolling bearings. Chinese Journal of Mechanical Engineering, 2016, 29(1): 204-211.

[6] Y Lei, F Jia, J Lin, et al. An intelligent fault diagnosis method using unsupervised feature learning towards mechanical big data. IEEE Transactions on Industrial Electronics, 2016, 63(5): 3137-3147. 
[7] M Zhang, J Tang, X Zhang, et al. Intelligent diagnosis of short hydraulic signal based on improved EEMD and SVM with few low-dimensional training samples. Chinese Journal of Mechanical Engineering, 2016, 29(2): 396-405.

[8] C Chen, B Zhang, G Vachtsevanos. Prediction of machine health condition using neuro-fuzzy and Bayesian algorithms. IEEE Transactions on Instrumentation and Measurement, 2012, 61(2): 297-306.

[9] B Scho “lkopf, J Platt, T Hofmann. Greedy layer-wise training of deep networks. Advances in Neural Information Processing Systems, 2007, 19: 153-160.

[10] DU W, TAO J, LI Y, et al. Wavelet leaders multifractal features based fault diagnosis of rotating mechanism [J]. Mechanical Systems \& Signal Processing, 2014, 43(1): 57-75.

[11] L Deng, G Hinton, B Kingsbury. New types of deep neural network learning for speech recognition and related applications: An overview. Proceedings of the IEEE International Conference on Acoustics, Speech and Signal Processing (ICASSP), Vancouver, BC, Canada, May 26-31, 2013: 8599-8603.

[12] X Yang, R Yan, R X Gao. Induction motor fault diagnosis using multiple class feature selection. Proceedings of 2015 IEEE International Instrumentation and Measurement Technology Conference (I2MTC), Pisa, Italy, May 11-15, 2015: 256-260. 